\title{
Blockade of $\beta$-adrenergic signaling suppresses inflammasome and alleviates cardiac fibrosis
}

\author{
Shipeng Dang ${ }^{1,2 \#}$, Zhen-Ye Zhang ${ }^{2 \#}$, Ku-Lin Li $^{2}$, Jie Zheng ${ }^{2}$, Ling-Ling Qian ${ }^{2}$, Xiao-Yu Liu ${ }^{2}$, Ying Wu ${ }^{2}$, \\ Chang-Ying Zhang ${ }^{2}$, Xiao-Xi Zhao ${ }^{2}$, Zhi-Ming Yu ${ }^{2}$, Ru-Xing Wang ${ }^{2}$, Tingbo Jiang ${ }^{1}$ \\ ${ }^{1}$ Department of Cardiology, The First Affiliated Hospital of Soochow University, Suzhou 215000, China; ${ }^{2}$ Department of Cardiology, The \\ Affiliated Wuxi People's Hospital of Nanjing Medical University, Wuxi 214000, China \\ Contributions: (I) Conception and design: S Dang, ZY Zhang; (II) Administrative support: ZM Yu, RX Wang, T Jiang; (III) Provision of study \\ materials or patients: S Dang, KL Li, J Zheng; (IV) Collection and assembly of data: LL Qian, XY Liu, Y Wu; (V) Data analysis and interpretation: \\ CY Zhang, XX Zhao; (VI) Manuscript writing: All authors; (VII) Final approval of manuscript: All authors. \\ \#These authors contributed equally to this work. \\ Correspondence to: Ru-Xing Wang, MD, PhD. Department of Cardiology, The Affiliated Wuxi People's Hospital of Nanjing Medical University, 299 \\ Qingyang Road, Wuxi 214000, China. Email: ruxingw@aliyun.com; Tingbo Jiang, MD, PhD. Department of Cardiology, First Affiliated Hospital of \\ Soochow University, Suzhou 215000, China. Email: 18906201122@189.cn.
}

\begin{abstract}
Background: Heart failure (HF) is an end-stage syndrome of all structural heart diseases which accompanies the loss of myocardium and cardiac fibrosis. Although the role of inflammasome in cardiac fibrosis has recently been a point of focus, the mechanism of inflammasome activation in HF has not yet been elucidated.

Methods: In this study, we investigated the expression of inflammasome proteins in a rat thoracic aorta constriction (TAC) model and cultured cardiac fibroblasts with stimulation of norepinephrine (NE).

Results: Our results showed that levels of inflammasome proteins in the myocardial of TAC rats were elevated. By blocking $\beta$-adrenergic signaling in the rats, inflammasome activation was suppressed and heart function was improved. The stimulation of cultured cardiac fibroblasts with NE activated inflammasome in vitro, which was abrogated by the inhibition of the calcium channels and reactive oxygen species (ROS). The activation of inflammasome by NE promoted cardiac fibrosis, whereas the inhibition of the calcium channels, ROS, and inflammasome reduced this effect.

Conclusions: The present study indicated that activation of inflammasome by $\beta$-adrenergic signaling promotes cardiac fibrosis. Therefore, modulation of inflammasome during HF might provide a novel strategy to treat this disease.
\end{abstract}

Keywords: Heart failure (HF); inflammasome; reactive oxygen species (ROS); $\beta$-adrenergic signaling; cardiac fibrosis

Submitted Dec 16, 2019. Accepted for publication Jan 14, 2020.

doi: 10.21037/atm.2020.02.31

View this article at: http://dx.doi.org/10.21037/atm.2020.02.31

\section{Introduction}

Heart failure (HF) is a major public health concern that threatens people's health and quality of life, affecting 1-2\% of the world's population (1). Characterized by the loss of myocardium and cardiac fibrosis, HF is a multifactorial, systemic disease (2). However, the molecular mechanisms underlying the pathological process of cardiac fibrosis remain poorly understood.

There is accumulating evidence that demonstrates the crucial role of inflammasome in cardiac fibrosis $(3,4)$. Inflammasome, a group of protein complexes, consists of several NOD-like receptors (NLR), such as NLRP3, NLRC4 and NLRP6, and an adaptor protein apoptosis-associated speck-like protein containing a caspase-recruitment domain (ASC) (5).The inflammasome responds to cellular infection 
or stress with the promotion of caspase- 1 and the generation of pro-inflammatory cytokines, including interleukin (IL)$1 \beta$ and IL-18. Operating as 'master molecules', these pro-inflammatory cytokines enhance the dysfunction of cardiomyocytes, subsequently leading to ventricular remodeling (6). It has also been reported that $\beta$-adrenergic signaling plays an important role in the regulation of autophagy, proliferation and apoptosis in cardiomyocytes (7). However, it remains unknown whether or not $\beta$-adrenergic signaling activates inflammasome and regulates the release of inflammatory factors.

$\beta$-adrenergic signaling is known to be overactivated during HF (8). The activation of the $\beta$-adrenergic signaling receptors leads to the conversion of adenosine triphosphate to the secondary messenger, cyclic adenosine monophosphate (cAMP). cAMP activates protein kinase A (PKA) and, subsequently, several $\mathrm{Ca}^{2+}$-cycling proteins, such as calcium channels, are phosphorylated by PKA (9). Activation of calcium channels mediates $\mathrm{Ca}^{2+}$ release, cardiac excitation-contraction coupling and calcium overload (10). Elevated cytosolic calcium activates the profibrotic pathway, leading to myocyte hypertrophy and fibrosis, as well as leading to mitochondria damage, which in turn generates reactive oxygen species (ROS) (11). ROS modulates the intracellular signaling pathway, promoting cell death and cardiac fibrosis.

In our study, we demonstrated that the activation of inflammasome by the $\beta$-adrenergic signaling played an important role in the enhancement of cardiac fibrosis. The mechanisms involved the regulation of calcium overload and generation of ROS.

\section{Methods}

\section{Transverse aortic constriction (TAC) model establishment and intervention}

Male Sprague-Dawley (SD) rats (180-200 g) were purchased from Jiangsu Institute of Schistosomiasis Control and Prevention in China. The rats were housed in a specific pathogen-free room (temperature $23 \pm 1{ }^{\circ} \mathrm{C}$ and humidity $55-$ $65 \%$ ) with access to standard food and water under regular 12-hour photoperiods. As was previously reported, a TAC model was established (12). Briefly, rats were anaesthetized in an induction chamber with $3 \%$ isoflurane mixed with $6 \mathrm{~L} / \mathrm{min} 100 \%$ oxygen, and anesthesia was maintained with $1.5 \%$ isoflurane. A temperature-controlled surgical table maintained their body temperature. A half way incision was made between two trachea cartilage rings, and the tracheal cannula was inserted into the trachea. The tracheal canula was connected to a rodent ventilator for maintaining a respiratory rate of 50 breaths $/ \mathrm{min}$, tidal volume of $2.0 \mathrm{~mL}$. The aortic arch was exposed by left anterior thoracotomy in the third intercostal region and separating the thymus and fat tissue. A small piece of a 6.0 silk suture was placed between the innominate and left carotid arteries. A $1.0 \mathrm{~mm}$ wire was placed parallel to the transverse aorta, after that the aortic arch was constricted to the diameter of the wire and then the wire was quickly removed. The sham control rats underwent an identical procedure but without TAC.

The rats were randomly divided into a metoprolol group and a TAC group to facilitate investigation into the effects of metoprolol treatment. For the metoprolol intervention group, metoprolol (sc-205751, Santa Cruz Biotechnology, Inc., TX, USA) was dissolved in disinfected water and administered $(1 \mathrm{mg} / \mathrm{kg} \times$ day $)$ orally to the TAC rats for the 8 weeks directly following the procedure. For the TAC group, the rats drank disinfected water normally. The rats were sacrificed 8 weeks after the TAC procedure, and their hearts were harvested and stored at $-80{ }^{\circ} \mathrm{C}$. The heart weight/body weight (HW/BW) ratios were calculated. Animal care was carried out according to the guidelines for the Principles of Laboratory Animal Care, and the protocol was approved by the Institutional Animal Care and Use Committee of The Affiliated Wuxi People's Hospital of Nanjing Medical University.

\section{Left ventricle ejection fraction measurement}

Echocardiography was used to evaluate cardiac function (ie33, Philip, Netherlands). Anesthesia induction of rats was performed with isoflurane and maintained with chloroform. $\mathrm{M}$-mode tracings were used to measure the left ventricular (LV) posterior wall end diastole (LVPWd), the LV internal dimension end diastole (LVIDd), the interventricular septum end diastole (IVSd), the LV ejection fraction (LVEF), and the max aortic pressure gradient (PG). Ascending and descending aortic flow velocities were measured by Doppler flow signals. All measurements were made by an observer who was blinded with respect to the identity of the tracings. All measurements were averaged over six consecutive cardiac cycles.

\section{Cardiac fibroblasts culture}

To isolate primary neonatal rat cardiac fibroblasts (NRCFs), 
neonatal SD rats (Jiangsu Institute of Schistosomiasis Control and Prevention in China) were sacrificed. Their hearts were quickly removed and digested with cardiomyocyte isolation enzyme 1 (88288, Thermo, USA) and cardiomyocyte isolation enzyme 2 (88289, Thermo, USA). Cells were cultured in DMEM to achieve primary cell isolation (88287, Thermo, USA) containing $10 \%$ fetal bovine serum (12664025, Gibco, USA), then seeded at $1 \times 10^{6} / 100-\mathrm{mm}$ dish (430167, Corning, NY, USA) and initially incubated at $37^{\circ} \mathrm{C}$ with $5 \% \mathrm{CO}_{2}$. After 2 hours, the unattached cells were discarded, and the attached cells were cultured further in DMEM containing 10\% FBS.

\section{siRNA transfection}

The NRCFs were placed in serum-free medium and subsequently transfected with NLRP3 siRNA (GenePharm, Shanghai, China) to inhibit the expression of NLRP3. The sequence of siRNA duplexes targeting NLRP3 were as follows: sense 5'-CCAGGAGAGAACUUCUUAUTT-3', anti-sense 5'-AUAAGAAGUUCUCUCCUGGTT-3'. The media containing siRNA was replaced by DMEM containing 10\% FBS 6 hours after transfection.

\section{Western blot}

Cells were lysed with ice-cold RIPA buffer (89900, Pierce, WI, USA) containing protease and phosphatase inhibitors (04693159001, Roche, Switzerland). The lysates were fractionated by SDS-PAGE and then transferred onto polyvinylidene difluoride membranes (GE Healthcare, USA). The PVDF membranes were incubated with primary antibodies for GAPDH (2118, Cell Signaling Technology, MA, USA), $\beta 1$-adrenergic receptor (ab3442, Abcam, England), Phospho-PKA (4781, Cell Signaling Technology, MA), NLRP3 (LS-C334192, LifeSpan BioSciences, USA), ASC2 (NBP1-78977, Novus Biologicals, CO, USA), cleaved caspase-1 (NB100-56565, Novus Biologicals, CO, USA), collagen I (ab34710, Abcam, England), and collagen III (ab7778, Abcam, England). ImageJ was used to quantify immunoblotting bands by densitometry. Densities were normalized to control treatment and relative folds were normalized to GAPDH.

\section{ROS assay}

The NRCFs were plated in 6-well plates and treated with NE $(2 \mu M)$ plus ROS inhibitor (N-acetyl cysteine, NAC,
A9165, Sigma-Aldrich, MO, USA) at $10 \mathrm{mM}$ for 30 minutes. Following this, the culture medium was removed, before the cells were washed with PBS and then incubated with CMH2DCFDA (C400, Invitrogen, USA) at a final concentration of $2.5 \mu \mathrm{M}$ in serum-free medium for 30 minutes at $37{ }^{\circ} \mathrm{C}$. The cells were washed with warmed PBS, removed from the plates, immediately resuspended in cold PBS containing $1 \% \mathrm{FBS}$, and subjected to flow cytometric analysis. A similar process was carried out for the unstained control cells, but all treatments and dyes were omitted.

\section{Cytosolic calcium concentration measurement}

Cytosolic calcium concentrations in the NRCFs were measured by LAMBDA DG-4 (Sutter Company, USA). The NRCFs were incubated with $5 \mu M$ Fura-2/AM and $\mathrm{L}$ type $\mathrm{Ca}^{2+}$ channel inhibitor verapamil (V4629, $1 \mu \mathrm{M}$, Sigma-Aldrich, MO) for 30 minutes at room temperature and were protected from light. After being loaded, the cells were washed, NE was added, and their fluorescence intensity ratios were recorded, with excitation wavelengths of $340 \mathrm{~nm}$ and $380 \mathrm{~nm}$, and an emission wavelength of $510 \mathrm{~nm}$. The fluorescence intensity ratios were calculated as F340/F380, and changes in the fluorescence intensity ratios were used to represent changes in cytosolic calcium concentrations.

\section{Histology}

Hematoxylinand eosin (H\&E) or Masson's trichrome were used to stain the paraffin-embedded 4- $\mu \mathrm{m} L V$ sections.

\section{Statistical analysis}

Quantitative data are presented as mean \pm standard error of the mean (SEM). Statistical analysis was performed by SPSS 17.0 software. Normality of data was checked, and non-parametric testing was carried out if the data was not normally distributed. An independent two tailed Student's $t$-test was used to assess significance, with $\mathrm{P}<0.05$ considered to be statistically significant.

\section{Results}

\section{Blockade of $\beta$-adrenergic signaling inbibited inflammasome in pressure-overload model}

A pressure overload model (TAC) was established to 

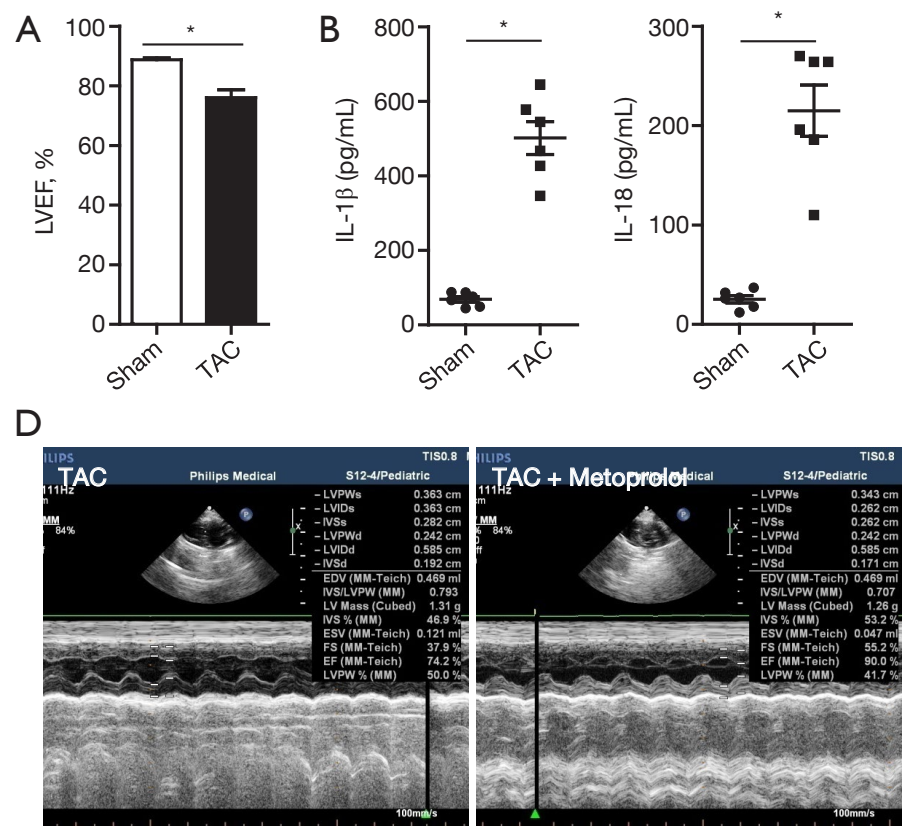

$\mathrm{F}$

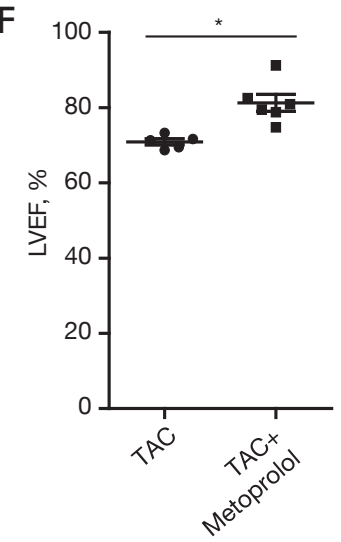

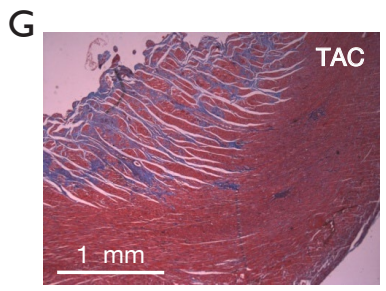

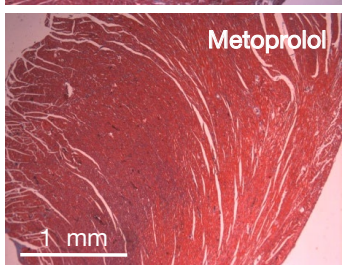

C
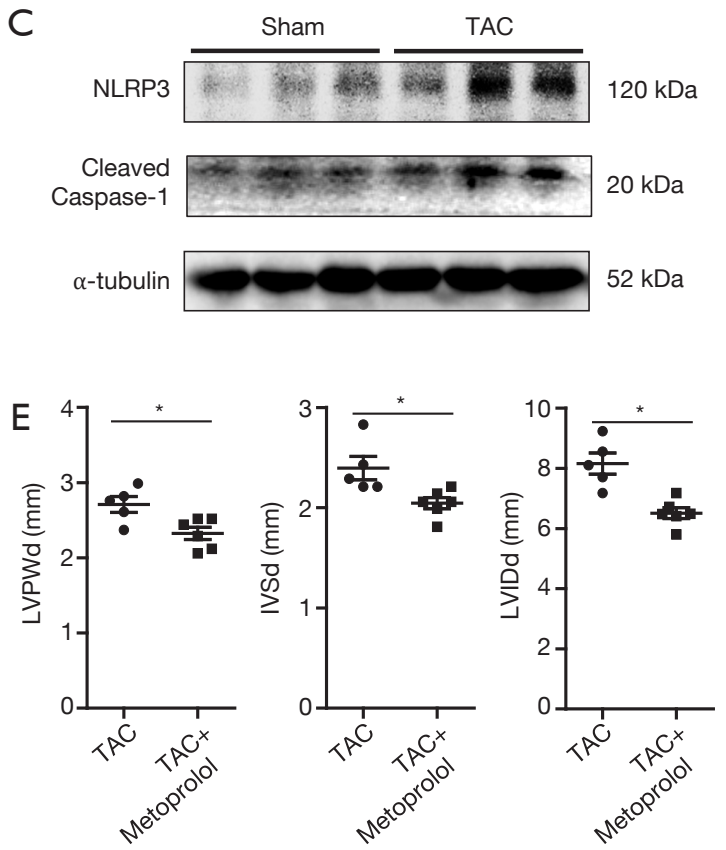

$\mathrm{H}$

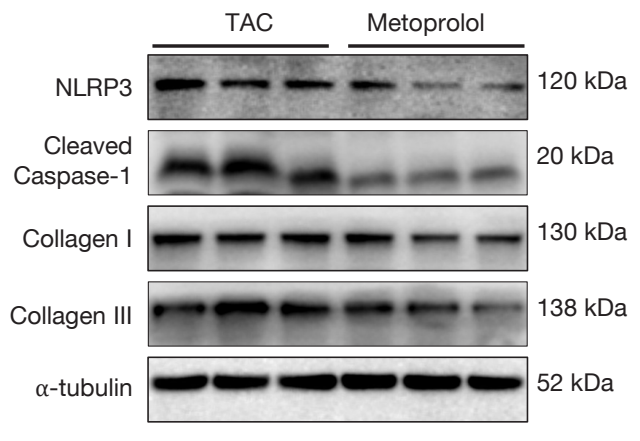

Figure 1 Blockade of $\beta$-adrenergic signaling improved heart function in pressure overload rats. (A) Two months after sham (n=14) or TAC $(\mathrm{n}=12)$ procedure, echocardiography was performed to measure LVEF; (B) serum IL-1 $\beta$ and IL-18 from sham and TAC rats were assayed with ELISA kits; (C) immunoblot analysis of LV myocardium from sham and TAC rat. TAC rats were treated with water (n=5) or water plus metoprolol $(\mathrm{n}=6)$ from the day of TAC procedure for 8 weeks; (D,E) echocardiography was performed to measure LVPWd, IVSd, LVIDd (D,E) and LVEF (F); (G) representative Masson-stain pictures of perivascular and myocardial fibrosis from each group; (H) immunoblot analysis of myocardium samples from the rats of TAC group and TAC treated with metoprolol group. ${ }^{*}, \mathrm{P}<0.05$. TAC, thoracic aorta constriction; LVEF, left ventricular ejection fraction; LVPWd, left ventricular posterior wall end diastole; IVSd, interventricular septum end diastole; LVIDd, left ventricular internal dimension end diastole.

investigate the role inflammasome plays in the development of cardiac remodeling. Our data demonstrated that left ventricle hypotrophy (Figure S1) and reduction of LVEF (Figure 1A) were observed in the TAC group rats but not in the sham group rats, which suggested that constriction of the aortic arch could induce cardiac remodeling and
HF. The key effectors of inflammasome, IL-1 $\beta$ and IL18 , were also investigated, and we found that the levels of serum IL-1 $\beta$ and IL-18 in the TAC group were significantly higher than those in the sham group (Figure $1 B, \mathrm{P}<0.05$ ). In the TAC group, the expressions of NLRP3 and cleaved caspase-1 were also upregulated (Figure 1C). These results 
A
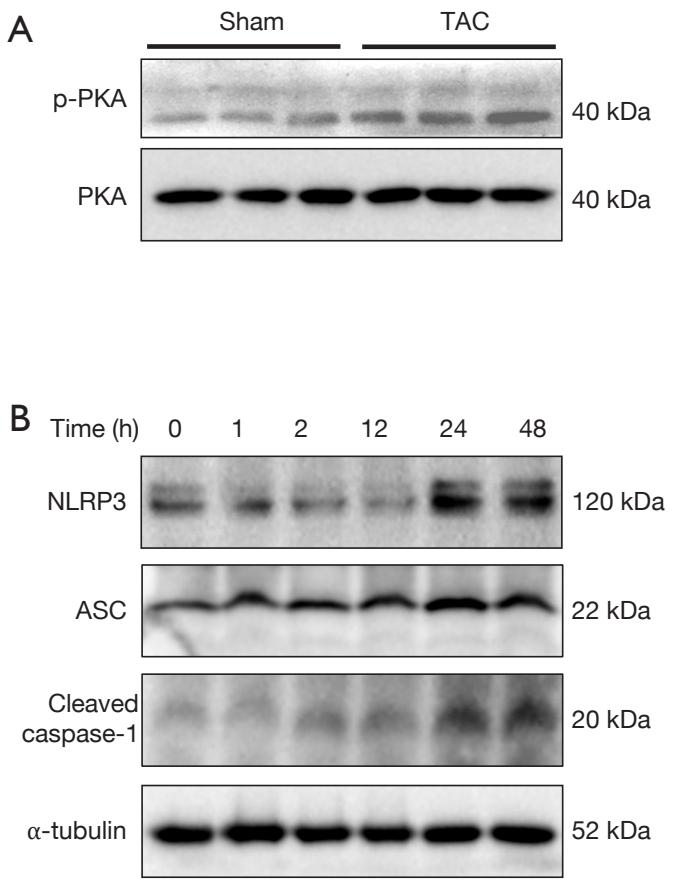
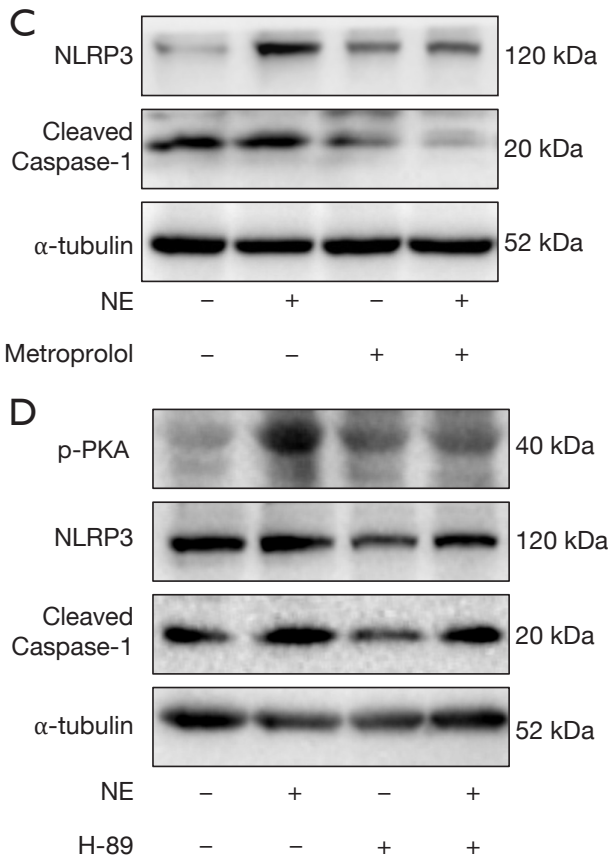

Figure 2 Inflammasome was activated by $\beta$-Adrenergic signaling. (A) PKA and pPKA were analyzed by immunoblot from the LV tissue of sham and TAC rats; (B) NRCFs were stimulated with NE for 1, 2, 12, 24 or 48 hours. NLRP3, ASC2 and cleaved caspase-1 were analyzed with immunoblot; (C,D) NRCFs were treated with $\beta$-adrenergic receptor blocker (Metoprolol) or PKA inhibitor (H-89) for 30 min prior to NE treatment and analyzed by immunoblots. PKA, protein kinase A; LV, left ventricular; TAC, thoracic aorta constriction; NRCF, neonatal rat cardiac fibroblast; NE, norepinephrine.

suggested that inflammasome was activated during pressureoverload mediated cardiac remodeling.

To determine whether the blockade of $\beta$-adrenergic signaling could reduce inflammasome and reverse cardiac fibrosis, we administered metoprolol orally to the TAC rats. As shown in Figure1D,E, LVPWd, IVSd and LVIDd were reduced in the metoprolol-treated TAC rats in comparison with the TAC rats that were not treated with metoprolol. The LVEFs were improved following metoprolol treatment (Figure $1 F$ ). Masson staining demonstrated reduced cardiac fibrosis in TAC rats treated with metoprolol (Figure $1 G$ ). In addition, the protein levels of NLRP3 and cleaved caspase-1 were lower in the LV myocardium of the TAC rats with metoprolol treatment than in the TAC rats (Figure $1 H$ ). Furthermore, the cardiac fibrosis proteins Collagen I and III were also reduced in the metoprolol-treated TAC rats treated in comparison with the TAC rats that did not receive metoprolol (Figure 1H). These data suggested that blockade of $\beta$-adrenergic signaling pathway reduced inflammasome and alleviated cardiac fibrosis.

\section{Inflammasome was activated in cardiac fibroblasts treated with the beta-adrenergic agonist, norepinephrine (NE)}

It was reported that catecholamine was elevated in patients who experienced $\mathrm{HF}$ and promoted the development of HF. We investigated the activation of the adrenergic signaling pathway in TAC model, and the results showed that the LV myocardium of the TAC rats had higher phosphorylation of PKA than those in the sham rats (Figure 2A). We then sought to investigate whether activation of the adrenergic signaling pathway could activate inflammasome. NRCFs were stimulated with NE for different periods of time. The results showed that expressions of NLRP3, ASC2 and cleaved caspase- 1 increased remarkably 24 hours after NE stimulation (Figure 2B). NRCFs were then treated with NE and the adrenergic receptor blocker metoprolol. We found that metoprolol abrogated the NE-induced up-regulation of NLRP3 and cleaved caspase-1 (Figure 2C). To explore whether the adrenergic signaling pathway was involved in the activation of inflammasome, we blocked PKA with H-89, before NE treatment. As shown in Figure 2D, the NE- 

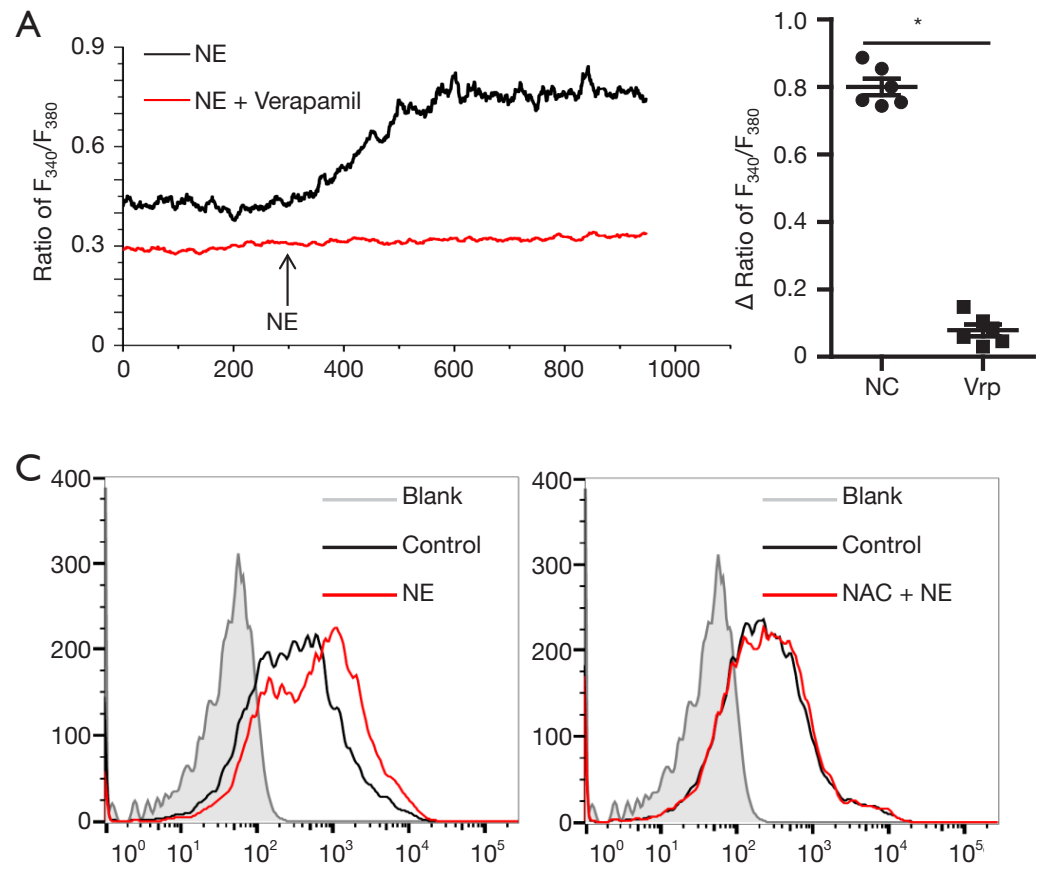

ROS

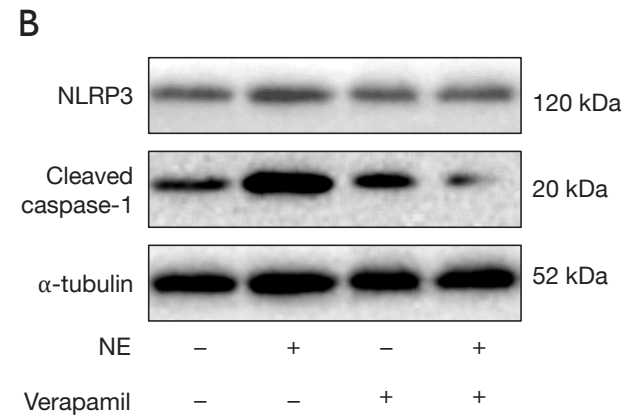

D

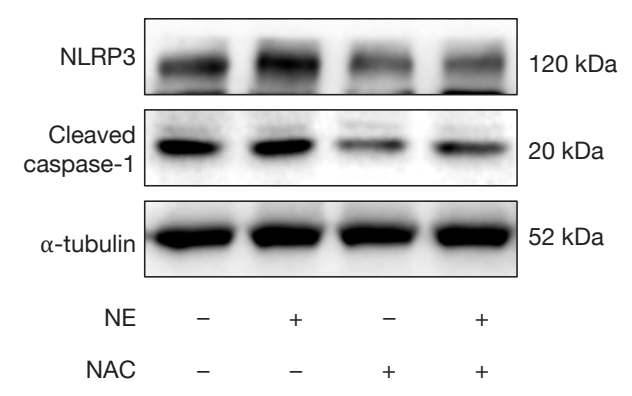

Figure 3 Inhibition of the calcium channel and ROS pathway inhibited inflammasome activation mediated by the adrenergic signaling. (A) NRCFs were incubated with Fura-2/AM for 30 minutes, and then treated with NE or with verapamil before NE stimulation. Fluorescence intensity ratios were recorded and ratios of F340/F380 were calculated; *, $\mathrm{P}<0.05$. (B) NRCFs were treated with verapamil for 30 min prior to NE treatment and analyzed by immunoblotting; (C) NRCFs were treated with or without NE for 48 h, then incubated with CM-H2DCFDA $(2.5 \mu M)$ for 30 min and analyzed by flow cytometry; (D) NRCFs were treated with ROS inhibitor (NAC) for 30 min prior to NE treatment and analyzed by immunoblotting. ROS, reactive oxygen species; NRCF, neonatal rat cardiac fibroblast; NE, norepinephrine; NAC, N-acetyl cysteine.

induced up-regulation of NLRP3 and cleaved caspase-1 was inhibited. These data suggest that NE activates inflammasome by adrenergic signaling.

\section{Adrenergic signaling activates inflammasome via the calcium channels/ROS}

To investigate how adrenergic signaling activates the inflammasome, we assayed calcium ion in cardiac fibroblasts. As shown in Figure $3 A$, cytosolic calcium increased in cardiac fibroblasts with stimulation of NE, while verapamil inhibited the increase of cytosolic calcium. Verapamil also suppressed the NE-induced up-regulation of NLRP3 and cleaved caspase-1 (Figure $3 B$ ). To investigate whether ROS was involved the activation of the inflammasome, the production of ROS in cardiac fibroblasts was examined. Our data showed that NE stimulation increased ROS production, whereas the blockade of the calcium channels suppressed ROS production in cardiac fibroblasts (Figure $3 C)$. Furthermore, the ROS inhibitor NAC reversed the upregulation of NLRP3 and cleaved caspase-1 which had been mediated by NE (Figure 3D). Overall, our data suggest that adrenergic signaling activates inflammasome via the calcium channel/ROS pathway.

\section{Inflammasome promoted cardiac fibrosis mediated by $\beta$-adrenergic signaling}

To investigate the role of inflammasome in cardiac fibrosis, NLRP3 was inhibited with RNAi in cardiac fibroblasts. Our data showed that protein level of NLRP3 was reduced following siRNA transfection, and its up-regulation through NE stimulation was inhibited. Moreover, the up-regulation of collagen I and collagen III induced by NE was also suppressed (Figure 4A). Next, to determine whether NE promotes cardiac fibrosis via $\beta$-adrenergic signaling, the adrenergic signaling 
A

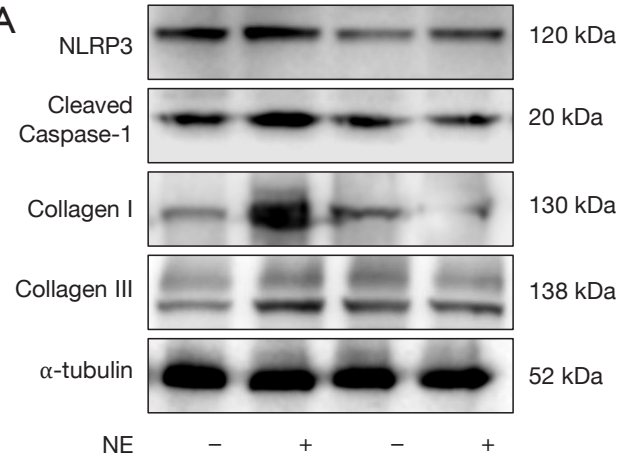

SiNLRP3

C

Collagen

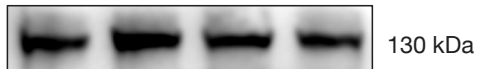

Collagen III

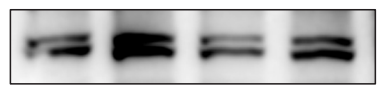

$\alpha$-tubulin

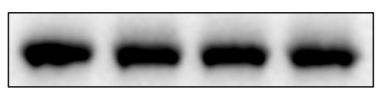

NE

$\mathrm{H}-89$

E

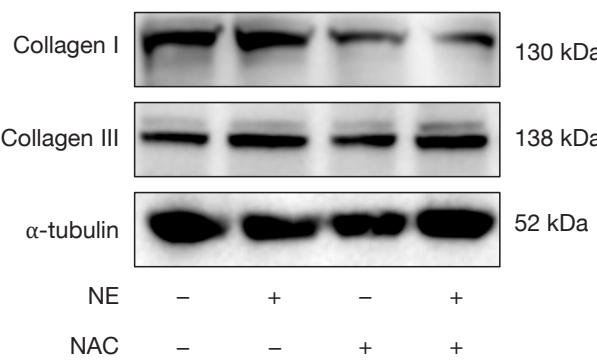

B

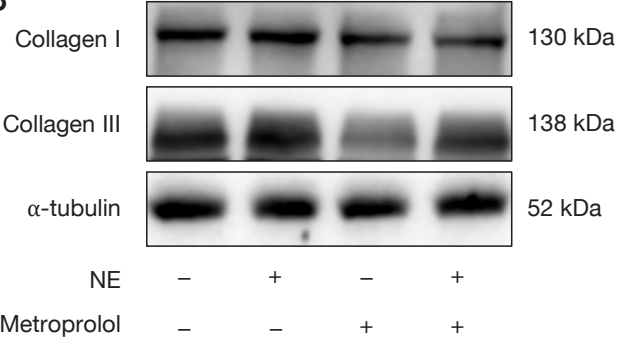

D

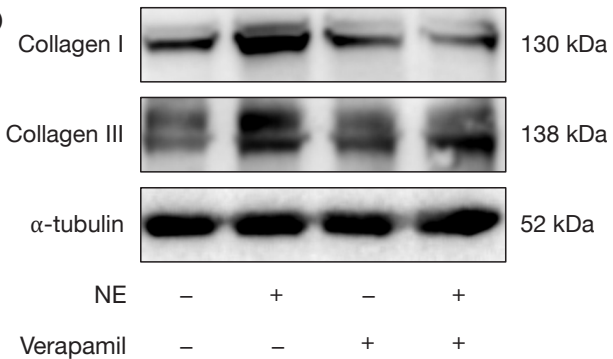

Figure 4 Activation of inflammasome via the $\beta$-adrenergic signaling promoted cardiac fibrosis. (A) NRCFs were transfected with siNLRP3 and treated with NE, then analyzed by immunoblots; (B) NRCFs were treated with metoprolol for 30 min prior to NE treatment and analyzed by immunoblotting; (C,D,E) NRCFs were treated with H-89 (C), verapamil (D) or NAC (E) for 30 min prior to NE treatment and analyzed by immunoblots. NRCF, neonatal rat cardiac fibroblast; NAC, N-acetyl cysteine; NE, norepinephrine.

was blocked with metoprolol. The results showed that the upregulation of collagen I and collagen III in the protein levels under NE stimulation was inhibited (Figure 4B). Furthermore, decreased expressions of collagen I and collagen III were detected by Western blotting when the PKA and calcium channels/ROS were inhibited, respectively (Figure 4C,D,E). Therefore, the NE-induced activation of inflammasome promoted cardiac fibrosis via $\beta$-adrenergic signaling, calcium channels, and the ROS pathway.

\section{Discussion}

Recently, HF has been reported to be associated with the loss of myocardium and cardiac fibrosis $(2,13)$; however, the underlying mechanisms of such a deterioration remain elusive. In this study, we found inflammasome was activated in HF rat cardiac fibroblasts and promoted cardiac fibrosis, while the blockade of adrenergic signaling inhibited inflammasome and reduced cardiac fibrosis. Mechanistic study showed that the activation of inflammasome by the adrenergic signaling promoted cardiac fibrosis via the calcium channel/ROS pathway. These findings suggested that adrenergic signaling/calcium channel/ROS/ inflammasome pathway might play an important role in the development of HF.

An increasing amount of evidence has demonstrated that 
the sympathetic nervous system plays a critical role in the development of cardiovascular diseases (14). Adrenergic receptors function as important regulators in cardiovascular disorders, particularly in HF (15). Adrenergic receptors belong to the family of guanine nucleotide-binding G protein-coupled receptors, and two classes have been identified: $\alpha$ and $\beta$ (16). The $\beta$-adrenergic receptors are abundantly expressed in the heart and $\beta$-adrenergic signaling plays an important role in the regulation of pathological process of cardiomyocytes (17). Moreover, the activation of $\beta$-adrenergic signaling has been reported to increase $\mathrm{Ca}^{2+}$ leakage via phosphorylating $\mathrm{Ca}^{2+}$-cycling proteins in response to the increase of secondary messenger cAMP (18). In this study, the phosphorylation of PKA was significantly higher in the TAC rat cardiac fibroblasts than in the sham rat cardiac fibroblasts, which indicates the activation of the adrenergic pathway in the pressureoverloaded rats. Furthermore, NE stimulation increased cytosolic calcium and cardiac fibrosis in cardiac fibroblasts, while inhibition of the calcium channels with verapamil decreased cardiac fibrosis. This indicated that $\beta$-adrenergic signaling was involved in the regulation of cardiac fibrosis in the TAC rat cardiac fibroblasts.

Inflammasome has been reported to be involved in regulation of cardiac fibrosis in HF (19-21). The NLRP3 inflammasome can be activated under stress and formed by NLRP3 and procaspase-1 via the adaptor protein ASC2 (22). Moreover, the NLRP3 inflammasome exerts an inflammatory effect through its regulation of the release of proinflammatory cytokines, including IL- $1 \beta$ and IL-18, which contribute to the enhancement of cardiomyocyte dysfunction and lead to ventricular remodeling and HF $(23,24)$. In this study, serum levels of IL- $1 \beta$ and IL-18 were significantly higher in the TAC rats. The TAC rat myocardium had a higher expression of NLRP3. Further examination revealed that NLRP3 inflammasome was activated in cardiac fibroblasts by NE. The knockdown of NLRP3 inhibited the induction of cardiac fibrosis by NE. Therefore, inflammasome functions as an important regulator in the pathological process of cardiac fibrosis during HF.

Recently, a growing number of studies have provided evidence that ROS generation notably contributes to HF (25). Under pathophysiological conditions, ROS overproduction is responsible for the activation of various signaling pathways and cell damage. Moreover, excessive oxidative stress has been reported to participate in the pathophysiology development of HF (26). Our data demonstrated that the inhibition of ROS reduced the expressions of NLRP3 and cleaved caspase-1, finally reversing cardiac fibrosis. This outcome suggested that excessive oxidative stress during HF contributed to the aggravation of NLRP3-inflammasome-induced cardiac fibrosis.

In conclusion, we demonstrated that the blockade of $\beta$-adrenergic signaling elevates heart function with reduction of cardiac fibrosis and activation of inflammasome by the $\beta$-adrenergic signaling plays an important role in the promotion of cardiac fibrosis via the calcium channel/ROS pathway. Thus, the modulation of $\beta$-adrenergic signaling, ROS, and inflammasome may provide a novel strategy for the future treatment of HF.

\section{Acknowledgments}

The authors wish to thank the technical staffs from the Center of Clinical Research at our hospital for their help. In addition, we would like to acknowledge Doctor Juan Lu for her help with measurement of rat heart function.

Funding: This work was supported by the National Natural Science Foundation of China (81400297, 81770331, 81500249 and 81500323) and Jiangsu Province Young Medical Talents (QNRC2016185).

\section{Footnote}

Conflicts of Interest: The authors have no conflicts of interest to declare.

Ethical Statement: The authors are accountable for all aspects of the work in ensuring that questions related to the accuracy or integrity of any part of the work are appropriately investigated and resolved. Animal care was carried out according to the guidelines for the Principles of Laboratory Animal Care, and the protocol was approved by the Institutional Animal Care and Use Committee of The Affiliated Wuxi People's Hospital of Nanjing Medical University.

\section{References}

1. Tanai E, Frantz S. Pathophysiology of Heart Failure. Compr Physiol 2015;6:187-214.

2. Parcha V, Arora P. Glycosylation of natriuretic peptides in obese heart failure: mechanistic insights. Ann Transl Med 2019;7:611. 
3. Butts B, Gary RA, Dunbar SB, et al. The Importance of NLRP3 Inflammasome in Heart Failure. J Card Fail 2015;21:586-93.

4. Butts B, Butler J, Dunbar SB, et al. ASC Methylation and Interleukin-1beta Are Associated with Aerobic Capacity in Heart Failure. Med Sci Sports Exerc 2017;49:1072-8.

5. Strowig T, Henao-Mejia J, Elinav E, et al. Inflammasomes in health and disease. Nature 2012;481:278-86.

6. Toldo S, Mezzaroma E, Mauro AG, et al. The inflammasome in myocardial injury and cardiac remodeling. Antioxid Redox Signal 2015;22:1146-61.

7. Imoto K, Hirakawa M, Okada M, et al. Canstatin modulates L-type calcium channel activity in rat ventricular cardiomyocytes. Biochem Biophys Res Commun 2018;499:954-9.

8. Floras JS, Ponikowski P. The sympathetic/parasympathetic imbalance in heart failure with reduced ejection fraction. Eur Heart J 2015;36:1974-82b.

9. Timofeyev V, Myers RE, Kim HJ, et al. Adenylyl cyclase subtype-specific compartmentalization: differential regulation of $\mathrm{L}$-type $\mathrm{Ca} 2+$ current in ventricular myocytes. Circ Res 2013;112:1567-76.

10. Fowler ED, Kong CHT, Hancox JC, et al. Late $\mathrm{Ca}(2+)$ Sparks and Ripples During the Systolic $\mathrm{Ca}(2+)$ Transient in Heart Muscle Cells. Circ Res 2018;122:473-8.

11. Denham NC, Pearman CM, Caldwell JL, et al. Calcium in the Pathophysiology of Atrial Fibrillation and Heart Failure. Front Physiol 2018;9:1380.

12. Gs AK, Raj B, Santhosh KS, et al. Ascending aortic constriction in rats for creation of pressure overload cardiac hypertrophy model. J Vis Exp 2014:e50983.

13. Yang $W, W u Z$, Yang K, et al. BMI1 Promotes Cardiac Fibrosis in Ischemia-Induced Heart Failure via the PTENPI3K/AKT-mTOR Signaling Pathway. Am J Physiol Heart Circ Physiol 2019;316:H61-9.

14. Santulli G, Iaccarino G. Adrenergic signaling in heart failure and cardiovascular aging. Maturitas 2016;93:65-72.

15. Zhang DY, Anderson AS. The sympathetic nervous system and heart failure. Cardiol Clin 2014;32:33-45.

16. Vicco MH, Pujato N, Bontempi I, et al. beta1-selective

Cite this article as: Dang S, Zhang ZY, Li KL, Zheng J, Qian LL, Liu XY, Wu Y, Zhang CY, Zhao XX, Yu ZM, Wang RX, Jiang $T$. Blockade of $\beta$-adrenergic signaling suppresses inflammasome and alleviates cardiac fibrosis. Ann Transl Med 2020;8(4):127. doi: 10.21037/atm.2020.02.31 adrenoceptor antagonists increase plasma levels of antip2 beta antibodies and decrease cardiac involvement in chronic progressive Chagas heart disease. Can J Cardiol 2014;30:332-7.

17. Negroni JA, Morotti S, Lascano EC, et al. beta-adrenergic effects on cardiac myofilaments and contraction in an integrated rabbit ventricular myocyte model. J Mol Cell Cardiol 2015;81:162-75.

18. Pereira L, Bare DJ, Galice S, et al. beta-Adrenergic induced SR $\mathrm{Ca}(2+)$ leak is mediated by an Epac-NOS pathway. J Mol Cell Cardiol 2017;108:8-16.

19. Zhou W, Chen C, Chen Z, et al. NLRP3: A Novel Mediator in Cardiovascular Disease. J Immunol Res 2018;2018:5702103.

20. Lim GB. Heart failure: Clonal haematopoiesis, IL-1beta, and the NLRP3 inflammasome in HF. Nat Rev Cardiol 2018;15:198.

21. Sano S, Oshima K, Wang Y, et al. Tet2-Mediated Clonal Hematopoiesis Accelerates Heart Failure Through a Mechanism Involving the IL-1beta/NLRP3 Inflammasome. J Am Coll Cardiol 2018;71:875-86.

22. Gan W, Ren J, Li T, et al. The SGK1 inhibitor EMD638683, prevents Angiotensin II-induced cardiac inflammation and fibrosis by blocking NLRP3 inflammasome activation. Biochim Biophys Acta Mol Basis Dis 2018;1864:1-10.

23. Van Tassell BW, Raleigh JM, Abbate A. Targeting interleukin-1 in heart failure and inflammatory heart disease. Curr Heart Fail Rep 2015;12:33-41.

24. Smykiewicz P, Segiet A, Keag M, et al. Proinflammatory cytokines and ageing of the cardiovascular-renal system. Mech Ageing Dev 2018;175:35-45.

25. van der Pol A, van Gilst WH, Voors AA, et al. Treating oxidative stress in heart failure: past, present and future. Eur J Heart Fail 2019;21:425-35.

26. Kiyuna LA, Albuquerque RPE, Chen CH, et al. Targeting mitochondrial dysfunction and oxidative stress in heart failure: Challenges and opportunities. Free Radic Biol Med 2018;129:155-68. 

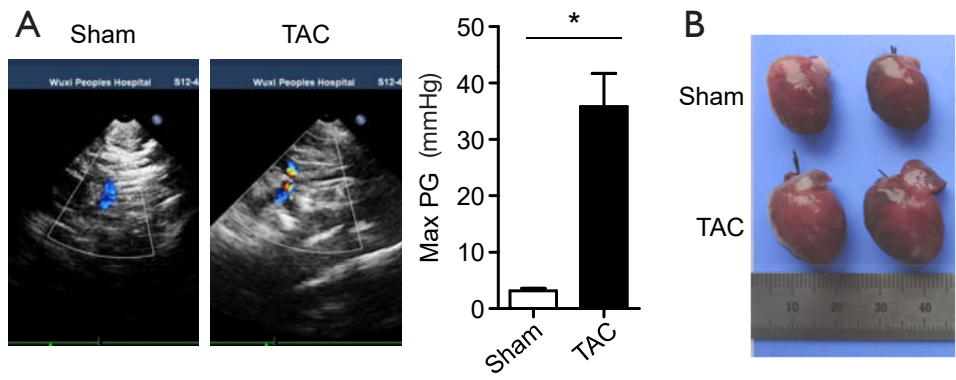

D
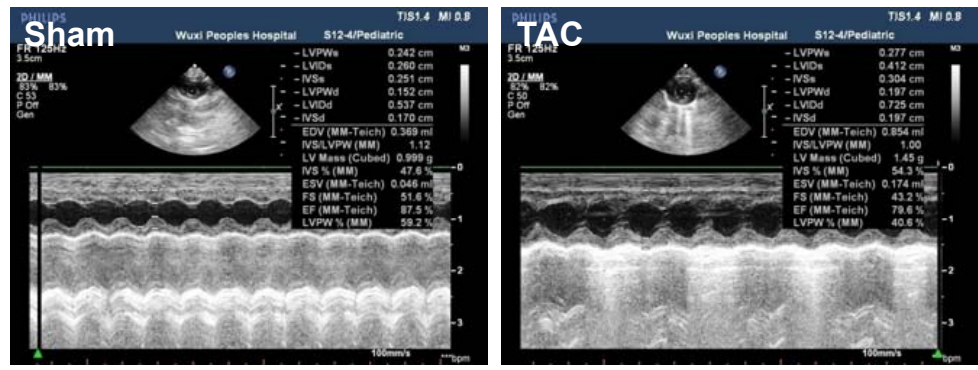

C

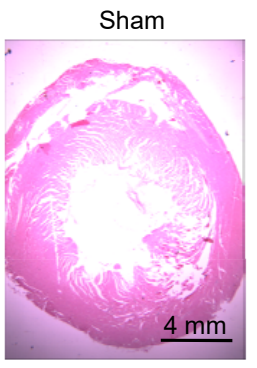

$\mathrm{E}$

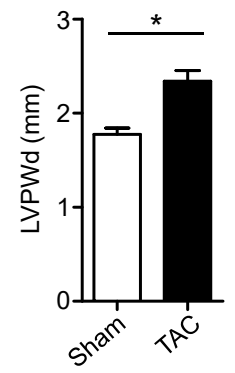

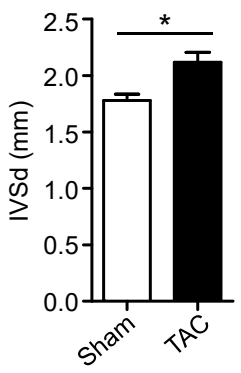

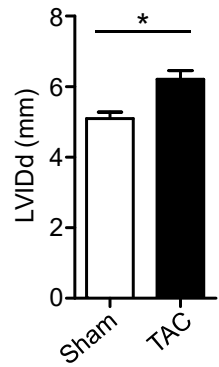

Figure S1 Pressure overload induced left ventricle hypotrophy. (A) One week after sham ( $\mathrm{n}=14)$ or TAC $(\mathrm{n}=12)$ procedure, echocardiography was performed to measure the PG of the aortic arch. Representative images and data are shown; $(\mathrm{B}, \mathrm{C})$ hearts from sham and TAC rats were harvested and imaged eight weeks after the procedure; (B) representative images and HW/BW are shown; (C) representative heart sections from these rats were stained with HE; (D,E) echocardiography was performed to evaluate LVPWd, IVSd, LVIDd of the LV. *, P<0.05. LVPWd, left ventricular posterior wall end diastole; IVSd, interventricular septum end diastole; LVIDd, left ventricular internal dimension end diastole; $L V$, left ventricular. 